\title{
Pacific
}

Journal of

Mathematics

\section{CONTRACTIVE ZERO-DIVISORS IN BERGMAN SPACES}

Peter Larkin Duren, DMitry KHAVINSON, Harold SEymour Shapiro and CARL Sundberg 


\title{
CONTRACTIVE ZERO-DIVISORS IN BERGMAN SPACES
}

\author{
Peter Duren, Dmitry Khavinson, Harold S. Shapiro \\ AND CARL SundBERG
}

\begin{abstract}
Generalizing a recent result of $\mathrm{H}$. Hedenmalm for $p=2$, a contractive zero-divisor is found in the Bergman space $A^{p}$ over the unit disk for $1 \leq p<\infty$. This is a function $G \in A^{p}$ with $\|G\|_{p}=1$ and a prescribed zero-set $\left\{\zeta_{j}\right\}$, uniquely determined by the contractive property $\|f / G\|_{p} \leq\|f\|_{p}$ for all $f \in A^{p}$ which vanish on $\left\{\zeta_{j}\right\}$. The proof uses the positivity of the biharmonic Green function of the disk. For a finite zero-set, the canonical divisor $G$ is represented explicitly in terms of the Bergman kernel of a certain weighted $A^{2}$ space. It is then shown that $G$ has an analytic continuation to a larger disk.
\end{abstract}

0. Introduction. It is well known that the zero-sets $\left\{\zeta_{j}\right\}$ of functions $f$ in the Hardy space $H^{p}$ are characterized by the Blaschke condition $\sum\left(1-\left|\zeta_{j}\right|\right)<\infty$, which guarantees the convergence of the Blaschke product

$$
B(z)=\prod_{j} b\left(z, \zeta_{j}\right), \quad \text { where } b(z, \zeta)=\frac{|\zeta|}{\zeta} \frac{\zeta-z}{1-\bar{\zeta} z}
$$

if $\zeta \neq 0$, and $b(z, 0)=z$. A basic theorem of F. Riesz (see [3], Ch. 2) asserts that $f / B$ is a nonvanishing function in $H^{p}$ with norm equal to that of $f$. This simple fact plays an important role in the theory of $H^{p}$ spaces, and it would be desirable to find an analogue for the Bergman spaces. We shall see in this paper that while isometric zerodivisors are not available in the Bergman spaces, there is an essentially unique contractive divisor of unit norm associated with every zero-set.

A function $f$ analytic in the unit disk $\mathbb{D}$ is said to belong to the Bergman space $A^{p}$, where $0<p<\infty$, if

$$
\|f\|_{p}^{p}=\iint_{\mathbb{D}}|f(z)|^{p} d \sigma<\infty .
$$

Here $d \sigma$ denotes the normalized element of area: $d \sigma=\frac{1}{\pi} d x d y$. For $1 \leq p<\infty$, the Bergman kernel $K(z, \zeta)=(1-\bar{\zeta} z)^{-2}$ has the reproducing property

$$
f(z)=\iint_{\mathbb{D}} f(\zeta) K(z, \zeta) d \sigma, \quad z \in \mathbb{D},
$$


for every $f \in A^{p}$. Bergman spaces $A^{p}(\Omega)$ can be defined more generally over any domain $\Omega \subset \mathbb{C}$ with finite area.

For the Bergman space $A^{2}$ over the disk Håkan Hedenmalm [8] recently constructed a canonical contractive zero-divisor which plays the role of a Blaschke product. This is a function $G \in A^{2}$ of unit norm, depending only on the zero-set of a given function $f \in A^{2}$, such that $f / G$ is a nonvanishing function in $A^{2}$ and $\|f / G\|_{2} \leq\|f\|_{2}$. Hedenmalm's idea was to construct $G$ as the unique solution to an extremal problem analogous to the one that produces Blaschke products in the Hardy spaces. The details will be described below.

Another analogue of a Blaschke product, valid more generally in $A^{p}$, was found earlier by Charles Horowitz [9]. For a zero-set $\left\{\zeta_{j}\right\}$ of a function $f \in A^{p}$, with repetitions according to multiplicity, Horowitz showed that the product

$$
B(z)=\prod_{j} b\left(z, \zeta_{j}\right)\left[2-b\left(z, \zeta_{j}\right)\right]
$$

converges. For $0<p<\infty$, he then proved that $f / B \in A^{p}$ and $\|f / B\|_{p} \leq C_{p}\|f\|_{p}$, where $C_{p}$ is a constant depending only on $p$. However, Horowitz' function $B$ need not belong to the space $A^{p}$. Horowitz [9] used this construction to show that every subset of an $A^{p}$ zero-set is an $A^{p}$ zero-set, for $0<p<\infty$.

In this paper we present a simplified version of Hedenmalm's construction while extending it to $A^{p}$ spaces over the disk with $1 \leq p<$ $\infty$. In fact, the argument will extend to $A^{p}(\Omega)$ for certain domains $\Omega$ with smooth boundary. One key to the more general result is to replace an explicit construction of Hedenmalm's, apparently viable only for $A^{2}$ of the disk, by a more flexible argument. The essential hypothesis is then that the biharmonic Green function of $\Omega$ be positive.

In order to make our presentation self-contained, we shall redevelop some of Hedenmalm's ideas in a more general context. There is inevitably some repetition, but we hope our discussion will help to bring the essential points into focus. Some parts of Hedenmalm's argument require totally different proofs for $p \neq 2$. For instance, the canonical divisor of a finite zero-set still has an analytic continuation to a larger disk, but the proof for $p \neq 2$ is much more difficult. For this reason the proof is deferred to the end of the paper, although some mild regularity at the boundary is needed earlier to establish the contractive property. In $\S 6$, the canonical divisor is represented in terms of the Bergman kernel function of a certain weighted $A^{2}$ space, which is then shown to have the desired analytic continuation. In $\S 5$, the canonical 
divisor for a single point is calculated explicitly, and its properties are used (following Hedenmalm's argument for $A^{2}$ ) to show in general that a canonical divisor can have no extraneous zeros. It is pointed out in $\S 7$ that a canonical divisor is always properly contractive.

Acknowledgment. We wish to thank Håkan Hedenmalm for stimulating discussions while this work was in progress, and for sharing some of his unpublished work with us.

1. The canonical divisor. Assume now that $1 \leq p<\infty$, and let $\left\{\zeta_{1}, \zeta_{2}, \ldots\right\}$ be the sequence of zeros (repeated according to multiplicity) of some function in $A^{p}$, where $\zeta_{j} \in \mathbb{D}$ and $\zeta_{j} \neq 0$. Let $N^{p}$ be the set of all $f \in A^{p}$ which vanish to (at least) the prescribed order at each point $\zeta_{j}$. Consider the extremal problem of maximizing $|f(0)|$ among all $f \in N^{p}$ with $\|f\|_{p}=1$. In order to see that an extremal function exists and is unique up to rotation, we pass to the equivalent problem of minimizing $\|f\|_{p}$ among all $f \in N^{p}$ with $f(0)=1$. For $1<p<\infty$ this is a closed convex subset of the uniformly convex Banach space $A^{p}$, so a standard theorem of functional analysis (see $[2]$, p. $74 ;[10]$, p. $353 ;[16]$, p. 52) says that it contains a unique element of smallest norm. Let $G$ be the (unique) solution to the original extremal problem with $G(0)>0$. Thus $G \in N^{p}$ and $\|G\|_{p}=1$. This extremal function $G$ will be called the canonical divisor of the zero-set $\left\{\zeta_{j}\right\}$.

For $p=1$ a different argument is required to prove the existence and uniqueness of the canonical divisor. In the original form of the extremal problem, a normal family argument may be applied to produce an extremal function. Another proof of uniqueness, valid for $1 \leq p<\infty$, involves kernel functions of weighted Bergman spaces and is deferred to the end of the paper. (See $\S 6$, Corollary of Theorem 5.) Meanwhile, we shall speak of the canonical divisor even for $p=1$. It may be observed that our discussion actually applies to any normalized solution of the extremal problem.

We suppose first that the prescribed zero-set is finite. Then we assert that the canonical divisor $G$ extends smoothly to the closed disk. In fact, we shall prove at the end of the paper $(\S 6)$ that it has an analytic continuation to a larger disk. This is much easier to see (cf. Hedenmalm [8]) in the special case $p=2$; indeed, it then follows from the theory of reproducing kernels (see [14], p. 62) that $G(z)$ is equal to a constant plus a linear combination of the Bergman kernel functions $K\left(z, \zeta_{1}\right), K\left(z, \zeta_{2}\right), \ldots, K\left(z, \zeta_{n}\right)$ and certain of their derivatives, 
where $\left\{\zeta_{1}, \ldots, \zeta_{n}\right\}$ is the prescribed zero-set. Thus $G$ is certainly analytic in the closed disk if $p=2$.

By a simple variational argument, we can now derive a special orthogonality property of the extremal function $G$. Let $L_{h}^{p}$ denote the class of harmonic functions in $L^{p}$ of the disk.

LEMMA 1. For each finite zero-set, the canonical divisor $G$ has the property

$$
\iint_{\mathbb{D}}\left\{|G(z)|^{p}-1\right\} u(z) d \sigma=0,
$$

for every function $u \in L_{h}^{1}$.

Proof. Consider the equivalent extremal problem of minimizing $\|f\|_{p}$ among all $f \in A^{p}$ with the prescribed zeros $\zeta_{j}$ and $f(0)=1$. Let $H$ be the unique extremal function. Then $H=G / G(0)$. For any function $f \in A^{p}$ with $f(0)=0$, the function $H^{*}=H(1+\lambda f)$ is in contention for each $\lambda \in \mathbb{C}$, since it has the prescribed zeros $\zeta_{j}$ and it satisfies $H^{*}(0)=1$. Thus by the extremal property of $H$,

$$
\begin{aligned}
\|H\|_{p}^{p} & \leq\left\|H^{*}\right\|_{p}^{p}=\iint_{\mathbb{D}}|H|^{p}|1+\lambda f|^{p} d \sigma \\
& =\|H\|_{p}^{p}+p \operatorname{Re}\left\{\lambda \iint_{\mathbb{D}}|H|^{p} f d \sigma\right\}+O\left(\lambda^{2}\right)
\end{aligned}
$$

for small $\lambda$. Letting $\lambda \rightarrow 0$ along an arbitrary ray, we conclude that

$$
\iint_{\mathbb{D}}|G|^{p} f d \sigma=0
$$

for all $f \in A^{p}$ with $f(0)=0$. For arbitrary $f \in A^{p}$, we use the formula

$$
f(0)=\iint_{\mathbb{D}} f(z) d \sigma
$$

and the fact that $\|G\|_{p}=1$ to deduce from (2) that

$$
\iint_{\mathbb{D}}\left\{|G|^{p}-1\right\} f d \sigma=0 .
$$

Since $G$ is bounded, this actually holds for all $f \in A^{1}$. Taking the real part and recalling that the class $L_{h}^{1}$ is preserved under harmonic conjugation, we arrive at (1).

Condition (2) depends on the fact that $G$ is bounded. A more general consequence of the extremal property of $G$ is that

$$
\iint_{\mathbb{D}}|G|^{p-2} \bar{G} h d \sigma=0
$$


for all $h \in N^{p}$ with $h(0)=0$. This is proved in the same way, even for an infinite zero-set, by the variation $H^{*}=H+\lambda h$.

It is important to note that the converse is also true. In other words, the condition (4) actually characterizes the extremal function.

LemMA 2. Given an $A^{p}$ zero-set $\left\{\zeta_{j}\right\}$ for $1 \leq p<\infty$, let a function $g \in N^{p}$ satisfy

$$
\iint_{\mathbb{D}}|g|^{p-2} \bar{g} h d \sigma=0
$$

for all $h \in N^{p}$ with $h(0)=0$. Then $g$ is a constant multiple of the canonical divisor $G$ of the set $\left\{\zeta_{j}\right\}$.

The proof appeals to the following known theorem of functional analysis, or approximation theory (cf. [16], p. 55 for further information).

Theorem A. For $1 \leq p<\infty$, let $M$ be a closed subspace of $L^{p}$ of the disk. Suppose that a function $g \in L^{p}$ has the property (5) for all $h \in M$. Then $\|g\|_{p}=d(g, M)$, the distance from $g$ to $M$.

Note. For $1 \leq p<2$, the expression $|g|^{p-2} \bar{g}$ should be taken to vanish wherever $g$ does. It may also be written as $|g|^{p-1} \overline{\operatorname{sgn} g}$.

Proof of Theorem A. There is no loss of generality in assuming $\|g\|_{p}=1$. Let $F=|g|^{p-2} \bar{g}$. Then $F \in L^{q}$ and $\|F\|_{q}=1$, where $q=p /(p-1)$ is the conjugate index. Thus the condition (5) implies

$$
\|g\|_{p}=\iint_{\mathbb{D}} F g d \sigma=\iint_{\mathbb{D}} F(g+h) d \sigma \leq\|g+h\|_{p}\|F\|_{q}=\|g+h\|_{p}
$$

for all $h \in M$. This shows that $\|g\|_{p}=d(g, M)$.

Deduction of Lemma 2. Let $M$ be the subspace of functions $h \in N^{p}$ with $h(0)=0$. Let $g \in N^{p}$ satisfy (5) for all $h \in M$, and suppose that $g(0)=1$. Then Theorem A says that $g$ is the (unique) function $H \in N^{p}$ of minimal norm with $H(0)=1$. Thus $g$ is a constant multiple of $G$.

2. A boundary-value problem. Again let $G$ be the canonical divisor of a finite zero-set $\left\{\zeta_{j}\right\}$. It is still implicitly assumed (with proof deferred to $\S 6$ ) that $G$ has a smooth extension to $\mathbb{T}=\partial \mathbb{D}$ and is in fact analytic in $\overline{\mathbb{D}}$. Then the boundary-value problem

$$
\begin{cases}\Delta \varphi=|G|^{p}-1 & \text { in } \mathbb{D}, \\ \varphi(z)=0 & \text { on } \mathbb{T}\end{cases}
$$


has a unique solution $\varphi \in C^{2}(\overline{\mathbb{D}})$. Here $\Delta$ denotes the Laplacian.

LEMMA 3. If $G$ is the canonical divisor of a finite zero-set, then the solution $\varphi$ of the boundary-value problem (6) has the properties

$$
\begin{cases}\text { (a) } \frac{\partial \varphi}{\partial n}=0 & \text { on } \mathbb{T} \\ \text { (b) } \varphi(z)>0 & \text { in } \mathbb{D}\end{cases}
$$

where $\partial \varphi / \partial n$ is the outer normal derivative.

In the case $p=2$, Hedenmalm [8] was able to verify the properties (7) by first displaying $\varphi$ explicitly as a double sum in terms of the (known) structure of the extremal function $G$, then making direct calculations. For $p \neq 2$ no explicit formulas are available and a different approach is necessary. We shall use Green's formula (see e.g. Nehari [11], Ch. 1)

$$
\iint_{\mathbb{D}}(u \Delta v-v \Delta u) d A=\int_{\mathbb{T}}\left(u \frac{\partial v}{\partial n}-v \frac{\partial u}{\partial n}\right) d s,
$$

valid for any functions $u, v \in C^{2}(\overline{\mathbb{D}})$, where $d A$ and $d s$ denote the elements of area and arclength, respectively.

Proof of (7a). It suffices to show that

$$
\int_{\mathbb{T}} u \frac{\partial \varphi}{\partial n} d s=0
$$

for all $u \in C^{2}(\overline{\mathbb{D}})$ harmonic in $\mathbb{D}$. But by Green's formula,

$$
\begin{aligned}
& \int_{\mathbb{T}} u \frac{\partial \varphi}{\partial n} d s=\int_{\mathbb{T}}\left(u \frac{\partial \varphi}{\partial n}-\varphi \frac{\partial u}{\partial n}\right) d s \\
& \quad=\iint_{\mathbb{D}}(u \Delta \varphi-\varphi \Delta u) d A=\iint_{\mathbb{D}}\left(|G|^{p}-1\right) u d A=0,
\end{aligned}
$$

in view of the construction (6) of $\varphi$, the harmonicity of $u$, and Lemma 1.

Proof of (7b). Since $\varphi=\partial \varphi / \partial n=0$ on $\mathbb{T}$, the function $\varphi$ has the integral representation

$$
\varphi(\zeta)=\iint_{\mathbb{D}} \Gamma(z, \zeta) \Delta^{2} \varphi(z) d A, \quad \zeta \in \mathbb{D},
$$

where $\Gamma$ is the biharmonic Green function of $\mathbb{D}$, given by the formula (10) $\Gamma(z, \zeta)=\frac{1}{16 \pi}\left\{|z-\zeta|^{2} \log \left|\frac{z-\zeta}{1-\bar{\zeta} z}\right|^{2}+\left(1-|z|^{2}\right)\left(1-|\zeta|^{2}\right)\right\}$. 
(See Garabedian [7], p. 272.) It will be shown presently that $\Gamma(z, \zeta)>$ 0 for all $z, \zeta \in \mathbb{D}$. Since

$$
\begin{aligned}
\Delta^{2} \varphi & =\Delta(\Delta \varphi)=\Delta\left(|G|^{p}-1\right) \\
& =4 \frac{\partial^{2}|G|^{p}}{\partial z \partial \bar{z}}=p^{2}|G|^{p-2}\left|G^{\prime}\right|^{2}>0
\end{aligned}
$$

except for a finite number of points in $\mathbb{D}$, the representation formula (9) will then prove (7b).

It remains to verify that the biharmonic Green function (10) of the disk is positive. This is stated as an exercise in Garabedian's book ([7], p. 275), so we record a proof here. Observe that

$$
\left|\frac{1-\bar{\zeta} z}{z-\zeta}\right|^{2}=\frac{\left(1-|z|^{2}\right)\left(1-|\zeta|^{2}\right)}{|z-\zeta|^{2}}+1,
$$

so the property $\Gamma(z, \zeta)>0$ reduces to the simple inequality $\log x<$ $x-1$ for $x>1$. This concludes the proof of Lemma 3 .

Note that the argument applies in any simply connected Jordan domain $\Omega \subset \mathbb{C}$ with smooth boundary, provided only that the biharmonic Green function of $\Omega$ is positive and that the canonical divisor $G$ is sufficiently smooth at the boundary to allow the application of Green's formula. Unfortunately, the biharmonic Green function need not always be positive; Garabedian [6] showed that a sufficiently eccentric ellipse is already a counterexample. (See also [13].)

3. The expansive property. Having produced a function $\varphi$ with the properties (6) and (7), we can now show that the canonical divisor has an "expansive" property under multiplication which will imply the contractive property under division.

LemMA 4. For $1 \leq p<\infty$, let $G$ be the canonical divisor of a finite zero-set. Then

$$
\|G f\|_{p} \geq\|f\|_{p} \quad \text { for all } f \in A^{p}
$$

Proof. We again appeal to Green's formula (8). Taking $u=\varphi$, we find

$$
\iint_{\mathbb{D}} v \Delta \varphi d \sigma=\iint_{\mathbb{D}} \varphi \Delta v d \sigma
$$

since $\varphi=\partial \varphi / \partial n=0$ on $\mathbb{T}$. We now choose $v=|f|^{p}$, where $f$ is a polynomial (or any function analytic in $\overline{\mathbb{D}}$ ). Then

$$
\Delta\left(|f|^{p}\right)=p^{2}|f|^{p-2}\left|f^{\prime}\right|^{2} \geq 0 .
$$


Since $\Delta \varphi=|G|^{p}-1$ and $\varphi>0$ in $\mathbb{D}$, we conclude that

$$
\iint_{\mathbb{D}}\left(|G|^{p}-1\right)|f|^{p} d \sigma \geq 0
$$

which is the desired inequality (11) for polynomials $f$. The result for an arbitrary function $f \in A^{p}$ now follows after approximating it in norm by polynomials.

The expansive property (11) of the canonical divisor has an important consequence which will be used in deriving the contractive property.

LeMma 5. Let $G$ be the canonical divisor of a finite zero-set. Then $|G(z)| \geq 1$ for all $z \in \mathbb{T}$.

Proof. The following argument is adapted from Hedenmalm [8]. Given $z_{0} \in \mathbb{T}$, let $q(z)=\frac{1}{2}\left(z+z_{0}\right)$ and define $Q_{n}(z)=q(z)^{n} /\left\|q^{n}\right\|_{p}$. Then $\left\|Q_{n}\right\|=1$ and $Q_{n}(z) \rightarrow 0$ uniformly on each closed subset of $\overline{\mathbb{D}} \backslash\left\{z_{0}\right\}$ as $n \rightarrow \infty$, because $q$ has a peak point at $z_{0}$ and it is easily verified that $\left\|q^{n}\right\|_{p} \geq C n^{-2 / p}$ for large $n$, where $C$ is a positive constant independent of $n$. Since $G$ is continuous at $z_{0}$, it follows that

$$
\iint_{\mathbb{D}}\left|G Q_{n}\right|^{p} d \sigma \rightarrow\left|G\left(z_{0}\right)\right|^{p}
$$

But by (11),

$$
\iint_{\mathbb{D}}\left|G Q_{n}\right|^{p} d \sigma \geq \iint_{\mathbb{D}}\left|Q_{n}\right|^{p} d \sigma=1 .
$$

Hence $\left|G\left(z_{0}\right)\right| \geq 1$.

LEMMA 6. Each canonical divisor $G$ has the property $G(0)<1$.

Proof. Recall that $G(0)>0$ and $\|G\|_{p}=1$. But for any nonconstant function $f \in A^{p}$, the inequality $|f(0)|<\|f\|_{p}$ follows from the integral representation (3) and Hölder's inequality.

At this point it may be helpful to clarify the status of the lemmas given above. Lemmas 2 and 6 are fully proved. However, the proofs of Lemmas 3, 4, and 5 all proceeded under the assumption that the canonical divisor $G$ of a finite zero-set has a smooth extension to the boundary. Lemma 1 makes the tacit assumption that $G$ is bounded. At the end of the paper (in $\S 6$ ), these conditional lemmas will be put on firm footing with the proof that $G$ has an analytic extension to the closed disk. There the uniqueness of the canonical divisor for 
$p=1$ will also be proved. Meanwhile, in $\S 4$ we shall make an explicit calculation of the canonical divisor $G_{\alpha}$ corresponding to a single zero $\alpha$. The construction will depend only on Lemma 2 . It will then be evident by inspection that $G_{\alpha}$ is analytic in $\overline{\mathbb{D}}$, so all of the earlier lemmas will apply unconditionally with $G$ replaced by $G_{\alpha}$.

4. Impossibility of extraneous zeros. Recall that a canonical divisor $G$ was defined by an extremal property where it was required to have zeros of prescribed orders or higher at prescribed points in the disk. The following theorem asserts that it can have no additional zeros.

TheORem 1. For $1 \leq p<\infty$, let $G$ be the canonical divisor associated with an $A^{p}$ zero-set $\left\{\zeta_{j}\right\}$ in $\mathbb{D} \backslash\{0\}$, finite or infinite. Then the order of the zero of $G$ at each $\zeta_{j}$ is precisely equal to the multiplicity assigned to $\zeta_{j}$, and $G(z) \neq 0$ elsewhere in the disk.

Surprisingly, the main difficulty is to prove this theorem in the special case where the prescribed zero-set $\left\{\zeta_{j}\right\}$ reduces to a single point. In this case we shall now calculate the canonical divisor explicitly. It will be clear that it has no extraneous zeros.

For an arbitrary point $\alpha \in \mathbb{D}$, let $G_{\alpha}$ denote the canonical divisor with (at least) a simple zero at $\alpha$. In view of Lemma 2, the divisor $G_{\alpha}$ can be constructed up to normalization by producing a function $g \in A^{p}$ which vanishes at $\alpha$ and satisfies the condition (5) for all $h \in A^{p}$ with $h(\alpha)=h(0)=0$. First rewrite (5) in the equivalent form

$$
\iint_{\mathbb{D}}|g(z)|^{p-2} \overline{g(z)}\left(\frac{z-\alpha}{1-\bar{\alpha} z}\right) z h(z) d \sigma(z)=0
$$

for all $h \in A^{p}$. Now let $g$ have the structure

$$
g(z)=\frac{z-\alpha}{1-\bar{\alpha} z} f(z),
$$

where $f$ is analytic in $\overline{\mathbb{D}}$ and $f(z) \neq 0$ there. Insert (13) into (12) and use the substitution

$$
w=\frac{z-\alpha}{1-\bar{\alpha} z}, \quad z=\frac{w+\alpha}{1+\bar{\alpha} w}
$$

with

$$
d \sigma(z)=\frac{\left(1-|\alpha|^{2}\right)^{2}}{|1+\bar{\alpha} w|^{4}} d \sigma(w)
$$

to transform the resulting condition to

$$
\iint_{\mathbb{D}} \overline{\Phi(w)}|w|^{p} \frac{w+\alpha}{1+\bar{\alpha} w} \Psi(w) d \sigma(w)=0,
$$


where

$$
\Phi(w)=F(w)^{p / 2} \frac{1-|\alpha|^{2}}{(1+\bar{\alpha} w)^{2}}, \quad F(w)=f\left(\frac{w+\alpha}{1+\bar{\alpha} w}\right)
$$

and

$$
\Psi(w)=\Phi(w) H(w) / F(w), \quad H(w)=h\left(\frac{w+\alpha}{1+\bar{\alpha} w}\right)
$$

Here $\Psi$ is to be an arbitrary function in $A^{p}$. Let

$$
\Phi(w)=\sum_{n=0}^{\infty} c_{n} w^{n}
$$

and choose $\Psi(w)=(1+\bar{\alpha} w) w^{k}$ to calculate from (14) the relation

$$
(2 k+p+2) c_{k+1}+\bar{\alpha}(2 k+p+4) c_{k}=0, \quad k=0,1,2, \ldots
$$

This shows that

$$
c_{n}=(2 n+p+2)(-\bar{\alpha})^{n-1}, \quad n=0,1,2, \ldots,
$$

up to a constant multiple. Summation of the power series then leads to the expression

$$
\Phi(w)=-(1 / \bar{\alpha})(1+\bar{\alpha} w)^{-2}(p \bar{\alpha} w+p+2) .
$$

Combining this with (15) and (13), one concludes that the canonical divisor has the form

$$
G_{\alpha}(z)=C \frac{z-\alpha}{1-\bar{\alpha} z}\left\{1+\frac{p}{2}\left(1+\bar{\alpha} \frac{z-\alpha}{1-\bar{\alpha} z}\right)\right\}^{2 / p}
$$

where $C$ is a suitable constant. From the requirements that $\left\|G_{\alpha}\right\|_{p}=$ 1 and $G_{\alpha}(0)>0$, one calculates

$$
C=-\frac{\bar{\alpha}}{|\alpha|}\left[1+\frac{p}{2}\left(1-|\alpha|^{2}\right)\right]^{-1 / p} .
$$

It is clear by inspection of the formula (16) that $G_{\alpha}$ is analytic in $\overline{\mathbb{D}}$ and has only a simple zero at $\alpha$. A review of the above calculations shows that $g=G_{\alpha}$ must satisfy (12) for all $h \in A^{p}$.

K. Yu. Osipenko and Michael Stessin [12] have independently obtained the formula for $G_{\alpha}$. Their approach is somewhat different from ours. Both methods lead to a more general formula where a multiple zero is prescribed at the point $\alpha$. 
Proof of Theorem 1. Suppose on the contrary that $G$ has a zero at some point $\alpha \neq \zeta_{j}$ or that it has a zero of higher order than required at some point $\alpha=\zeta_{j}$. Let $G_{\alpha}$ be the canonical divisor of $\{\alpha\}$ as constructed above. Then the function $G / G_{\alpha}$ is analytic in $\mathbb{D}$ and has a zero of at least the prescribed order at each point $\zeta_{j}$. But $G_{\alpha}$ has a smooth extension to $\overline{\mathbb{D}}$, so the expansive property of multiplication by $G_{\alpha}$ (Lemma 4) is established unconditionally. This implies Lemma 5, so $\left|G_{\alpha}(z)\right| \geq 1$ on $\mathbb{T}$, a fact which can also be seen by inspection of the formula for $G_{\alpha}$. Thus since $G \in A^{p}$, it follows that $G / G_{\alpha} \in A^{p}$. On the other hand, Lemma 4 states that $\left\|G_{\alpha} f\right\|_{p} \geq\|f\|_{p}$ for all $f \in A^{p}$. Taking $f=G / G_{\alpha}$, we conclude that $\left\|G / G_{\alpha}\right\|_{p} \leq\|G\|_{p}=1$. Thus the function $G / G_{\alpha}$ has all of the properties required in the extremal problem through which $G$ was defined. However, Lemma 6 (or direct inspection) shows that $G_{\alpha}(0)<1$, so $G(0) / G_{\alpha}(0)>G(0)$, which violates the extremal property of $G$. This contradiction shows that $G$ can have no extraneous zeros.

It should be stressed that Theorem 1 has been established unconditionally. The proof made no appeal to the fact that the canonical divisor of an arbitrary finite zero-set has a smooth extension to the boundary.

5. The contractive property. It is now a short step to the main result, the contractive property of the canonical divisor.

Theorem 2. Let $1 \leq p<\infty$, let $\left\{\zeta_{j}\right\}$ be an $A^{p}$ zero-set in $\mathbb{D} \backslash\{0\}$, and let $G$ be the corresponding canonical divisor. Let $f \in A^{p}$ have a zero at each $\zeta_{j}$ of order no smaller than the prescribed multiplicity. Then $f / G \in A^{p}$ and $\|f / G\|_{p} \leq\|f\|_{p}$. Furthermore, $G$ is the only function in $A^{p}$, normalized so that $\|G\|_{p}=1$ and $G(0) \geq 0$, which has this contractive property for every function $f \in A^{p}$ whose zero-set contains $\left\{\zeta_{j}\right\}$.

The contractive property stated in this theorem is stronger than the expansive property given by (11). Moreover, it must be kept in mind that (11) was proved only for canonical divisors of finite zero-sets. In order to pass to infinite zero-sets, we need the following lemma.

LEMMA 7. Let $\left\{\zeta_{j}\right\}$ be an $A^{p}$ zero-set, and let $G$ be its canonical divisor. Let $G_{n}$ be the canonical divisor of the finite subset $\left\{\zeta_{1}, \ldots, \zeta_{n}\right\}$. Then $G_{n}(z) \rightarrow G(z)$ uniformly on each compact subset of $\mathbb{D}$ as $n \rightarrow$ $\infty$. Furthermore, $\left\|G_{n}-G\right\|_{p} \rightarrow 0$. 
Proof of lemma. Since $\left\|G_{n}\right\|_{p}=1$ for $n=1,2, \ldots$, it is clear that the family $\left\{G_{n}\right\}$ is locally bounded and is therefore a normal family. In other words, some subsequence converges uniformly on compact subsets to a function $H$ analytic in $\mathbb{D}$. According to Theorem 1 and Hurwitz' theorem, $H$ has precisely the zeros $\left\{\zeta_{j}\right\}$, with the prescribed multiplicities. By Fatou's lemma, $\|H\|_{p} \leq 1$. But $G_{n}(0) \geq G(0)$ by the extremal property of $G_{n}$, and so $H(0) \geq G(0)$. It now follows from the uniqueness of the extremal function $G$ that $H(0)=G(0)$ and $H=G$. Since the argument shows that every subsequence of $\left\{G_{n}\right\}$ has the same limit, it is clear that $G_{n}(z) \rightarrow G(z)$ uniformly on compact sets. (Here one may also invoke Vitali's theorem for normal families.) To show that $G_{n}$ tends in norm to $G$, one need only recall that in $L^{p}$ of any measure space $(0<p<\infty)$, the two conditions $f_{n}(x) \rightarrow f(x)$ a.e. and $\left\|f_{n}\right\|_{p} \rightarrow\|f\|_{p}$ imply that $\left\|f_{n}-f\right\|_{p} \rightarrow 0$.

Proof of theorem. In view of Theorem $1, f / G_{n}$ is analytic in $\mathbb{D}$. But $G_{n}$ is continuous in $\overline{\mathbb{D}}$ and by Lemma 5 it satisfies $\left|G_{n}(z)\right| \geq 1$ on $\mathbb{T}$. Thus $f / G_{n} \in A^{p}$. Since $G_{n}$ is the canonical divisor of a finite zeroset, the expansive property (11) may now be invoked to conclude that $\|f\|_{p}=\left\|G_{n}\left(f / G_{n}\right)\right\|_{p} \geq\left\|f / G_{n}\right\|_{p}$, which is the contractive property for finite zero-sets.

Now let $g_{n}=f / G_{n}$ and $g=f / G$. It was just observed that $g_{n} \in A^{p}$ and $\left\|g_{n}\right\|_{p} \leq\|f\|_{p}$. On the other hand, $g$ is analytic in $\mathbb{D}$, and Lemma 7 shows that $g_{n}(z) \rightarrow g(z)$ uniformly on compact sets. Thus Fatou's lemma gives $\|g\|_{p} \leq\|f\|_{p}$ and $g \in A^{p}$. This shows that $G$ is a contractive divisor.

To prove the uniqueness of the contractive divisor, let $H \in A^{p}$ be another function vanishing on $\left\{\zeta_{j}\right\}$ with the properties $\|H\|_{p}=1$, $H(0) \geq 0$, and $\|f / H\|_{p} \leq\|f\|_{p}$ for all $f \in A^{p}$ vanishing on $\left\{\zeta_{j}\right\}$. Then in particular the function $h=G / H \in A^{p}$ and $\|h\|_{p} \leq\|G\|_{p}=$ 1. Thus $H(0)>0$. It follows from Hölder's inequality, as in the proof of Lemma 6, that $h(0) \leq\|h\|_{p} \leq 1$, and $h(0)<1$ unless $h$ is constant. In other words, the assumption that $H \neq G$ implies that $H(0)>G(0)$, violating the extremal property of $G$. This proves the uniqueness and completes the proof of Theorem 2 .

As an immediate corollary, we may state the following stronger form of Lemma 4.

THEOREM 3. Let $\left\{\zeta_{j}\right\}$ be an $A^{p}$ zero-set, and let $G$ be its canonical divisor. Then $\|G f\|_{p} \geq\|f\|_{p}$ for all $f \in A^{p}$. 
Proof. If $G f \notin A^{p}$, there is nothing to prove. If $G f \in A^{p}$, then its zero-set contains $\left\{\zeta_{j}\right\}$ and Theorem 2 gives $\|f\|_{p}=\|(G f) / G\|_{p} \leq$ $\|G f\|_{p}$.

Theorems 1, 2, and 3 can all be generalized to arbitrary subsets of $A^{p}$ zero-sets, without a priori knowledge that they are themselves $A^{p}$ zero-sets. A consequence is the theorem of Horowitz [9] that every subset of an $A^{p}$ zero-set is an $A^{p}$ zero-set.

6. Regularity of the canonical divisor. Our final objective is to show that the canonical divisor of a finite zero-set has an analytic continuation beyond the boundary of the unit disk. For $p=2$ this is an obvious corollary of the fact that the canonical divisor is then a finite linear combination of Bergman kernel functions and their derivatives (cf. $\S 1$ and [8]). A simple argument then extends the result to the case where $p$ is any even integer. Indeed, the implication results from a general connection between the canonical divisors, which will now be stated as a lemma.

Lemma 8. Suppose $1 \leq q<\infty$, and let $p=m q$ for some integer $m \geq 2$. Let $G$ be the canonical divisor in $A^{p}$ of a finite zero-set $\left\{\zeta_{j}\right\}$, and let $H$ be the canonical divisor in $A^{q}$ of the zero-set $\left\{\omega_{j}\right\}$ obtained from $\left\{\zeta_{j}\right\}$ by including each $\zeta_{j}$ exactly $m$ times. Then $H=G^{m}$.

Proof. Let $F=G^{m}$ and observe that $\|F\|_{q}=1, F(0)>0$, and $F$ has the required zeros $\left\{\omega_{j}\right\}$. If $F \neq H$, then $H(0)>F(0)$ and so $H(0)^{1 / m}>G(0)$. But $H^{1 / m}$ is analytic and has all of the properties required in the extremal problem which determines $G$, so this contradicts the extremality of $G$. Since the uniqueness of the canonical divisor is not yet established for $q=1$, the lemma must meanwhile be interpreted to say in this case that $G^{m}$ is a canonical divisor in $A^{1}$.

With $q=2$, Lemma 8 allows the regularity of the canonical divisor in $A^{2 m}$ to be deduced from that of the canonical divisor in $A^{2}$, together with the fact that the latter has no zeros on the boundary (see Lemma 5). For arbitrary $p \quad(1 \leq p<\infty)$, the proof will proceed by expressing the canonical divisor in terms of the reproducing kernel of a certain weighted $A^{2}$ space. This representation is of independent interest and is stated below as Theorem 4 . The proof then reduces to showing that the kernel function has an analytic continuation.

The terminology must first be explained. For a weight-function $w(z) \geq 0$ in $\mathbb{D}$, the weighted Bergman space $A_{w}^{2}$ consists of those 
analytic functions $f$ for which

$$
\|f\|_{2, w}=\left\{\iint_{\mathbb{D}}|f(z)|^{2} w(z) d \sigma\right\}^{1 / 2}<\infty .
$$

The kernel function $J(z, \zeta)$ of $A_{w}^{2}$ is characterized by the reproducing property

$$
f(z)=\iint_{\mathbb{D}} f(\zeta) J(z, \zeta) w(\zeta) d \sigma, \quad f \in A_{w}^{2} .
$$

Here $J(\cdot, \zeta) \in A_{w}^{2}$ for each $\zeta \in \mathbb{D}$, and $\overline{J(z, \zeta)}=J(\zeta, z)$. The kernel function of a more general space $A_{w}^{2}(\Omega)$ over a region $\Omega \subset \mathbb{C}$ is defined in the same way and has the same properties.

THEOREM 4. In the space $A^{p} \quad(1 \leq p<\infty)$, let $G$ be the canonical divisor of a finite zero-set and let $B(z)$ be the corresponding finite Blaschke product. Then

$$
G(z)=J(0,0)^{-1 / p} B(z) J(z, 0)^{2 / p},
$$

where $J(z, \zeta)$ is the kernel function of the Bergman space $A_{w}^{2}$ with weight $w(z)=|B(z)|^{p}$. In particular, $J(z, 0) \neq 0$ in $\mathbb{D}$.

Proof. In view of Theorem 1 , the canonical divisor has the factorization $G(z)=B(z) k(z)^{2 / p}$, where $k \in A^{2}$ and $k(z) \neq 0$ in $\mathbb{D}$. Furthermore, $G$ has the property (4), which is equivalent to

$$
\iint_{\mathbb{D}}|G|^{p-2} \bar{G} B f d \sigma=f(0) \iint_{\mathbb{D}}|G|^{p-2} \bar{G} B d \sigma, \quad f \in A^{p},
$$

since each function $h \in N^{p}$ has the form $h=B f$ for some $f \in A^{p}$; and $f=f(0)+[f-f(0)]$. When the factorization of $G$ is inserted into (21), the condition reduces to

$$
\iint_{\mathbb{D}} k^{1-2 / p} \bar{k} f|B|^{p} d \sigma=f(0) \iint_{\mathbb{D}} k^{1-2 / p} \bar{k}|B|^{p} d \sigma, \quad f \in A^{p} .
$$

The integral on the right-hand side of (22) may be calculated from the fact that $\|G\|_{p}=1$. This property and (22) give

$$
1=\iint_{\mathbb{D}} k^{1-2 / p} k^{2 / p} \bar{k}|B|^{p} d \sigma=k(0)^{2 / p} \iint_{\mathbb{D}} k^{1-2 / p} \bar{k}|B|^{p} d \sigma,
$$

so that the condition (22) becomes

$$
\iint_{\mathbb{D}} k^{1-2 / p} f \bar{k}|B|^{p} d \sigma=k(0)^{-2 / p} f(0), \quad f \in A^{p} .
$$


Suppose now that $1 \leq p \leq 2$, and choose $f=Q k^{2 p-1}$, where $Q$ is a polynomial. Then $f \in A^{p}$ because $0 \leq 2-p<2$; and (23) shows that

$$
k(0) \iint_{\mathbb{D}} Q \bar{k}|B|^{p} d \sigma=Q(0)
$$

for all polynomials $Q$, hence for all $Q \in A_{w}^{2}$ with $w=|B|^{p}$. But (24) is a reproducing property, so $k(0) \overline{k(z)}$ is identified with the kernel function $J(0, z)$. Hence $k(z)=C J(z, 0)$ for some constant $C$. This gives the formula (19), where the constant is determined by the requirements that $\|G\|_{p}=1$ and $G(0)>0$.

Suppose next that $2<p<\infty$, and choose an integer $m \geq 2$ so that $q=p / m$ lies in the interval $1<q<2$. Given a finite zero-set, again let $G=B k^{2 / p}$ be its canonical divisor in $A^{p}$. Then Lemma 8 says that $G^{m}=B^{m} k^{2 / q}$ is the canonical divisor in $A^{q}$ of the zero-set with Blaschke product $B^{m}$. Because $1<q<2$, it follows from what has already been proved that $k(z)=C J(z, 0)$, where $J(z, \zeta)$ is the kernel function in the space $A_{w}^{2}$ with weight $w=\left|B^{m}\right|^{q}=|B|^{p}$. Thus the representation (19) is valid also for $2<p<\infty$, and the proof is complete.

Note that the nonvanishing of the kernel function $J(z, 0)$ has been established as a byproduct of the proof. Håkan Hedenmalm (private communication) found a direct and elegant proof of this remarkable fact, before we discovered the proof given above. If the kernel function is known a priori not to vanish in $\mathbb{D}$, an alternate proof of Theorem 4 can be given for $2 \leq p<\infty$ by showing that the function $G$ defined by (19) has the property (5) of Lemma 2, and is therefore the canonical divisor. Actually, it is a corollary of Theorems 4 and 5 that $J(z, 0) \neq 0$ in $\overline{\mathbb{D}}$. If this were known a priori, the whole proof of Theorem 4 (for $1 \leq p<\infty$ ) could be based on Lemma 2 .

The next lemma contains the crux of the analytic continuation argument. It is stated in a form more general than actually needed here. The result may be found in a paper of Garabedian [6], where a direct proof is based on integral representations. We offer a short proof which relies heavily, however, on the phenomenon of elliptic regularity.

LEMMA 9. Let $\Omega \subset \mathbb{C}$ be a Jordan domain with analytic boundary. Let $w(z) \geq 0$ be a weight-function which is real-analytic in $\bar{\Omega}$ and is bounded away from zero near $\partial \Omega$. Let $J(z, \zeta)$ be the kernel function 
of $A_{w}^{2}(\Omega)$. Then for each fixed $\zeta$ in $\Omega$, the function $J(z, \zeta)$ has an analytic continuation across $\partial \Omega$.

Proof. The kernel function $J(z, \zeta)$ has the reproducing property

$$
f(z)=\iint_{\Omega} f(\zeta) J(z, \zeta) w(\zeta) d \sigma, \quad f \in A_{w}^{2}(\Omega) .
$$

In other words, for each point $z_{0} \in \Omega$, the distribution

$$
u(\zeta)=\left[w\left(z_{0}\right) J\left(z_{0}, \zeta\right)-\delta_{z_{0}}(\zeta)\right] w(\zeta)
$$

annihilates $A_{w}^{2}(\Omega)$. But $A_{w}^{2}(\Omega)=A^{2}(\Omega)$ as sets, since $w$ is bounded away from zero and infinity near the boundary. Thus by Havin's lemma (see [17], p. 28), there exists a distribution $U=v+\psi$, where $v$ has compact support in $\Omega$ and $\psi$ belongs to the Sobolev space $W_{0}^{1,2}(\Omega)$, such that $u=\partial U / \partial \bar{\zeta}$. Therefore, the relation

$$
\frac{\partial \psi}{\partial \bar{\zeta}}=w\left(z_{0}\right) w(\zeta) J\left(z_{0}, \zeta\right)
$$

holds near $\partial \Omega$. But since $J\left(z_{0}, \zeta\right)$ is an anti-analytic function of $\zeta$, this implies that

$$
L \psi=\frac{\partial}{\partial \zeta}\left\{\frac{1}{w(\zeta)} \frac{\partial \psi}{\partial \bar{\zeta}}\right\}=0
$$

near $\partial \Omega$. Here $L$ is a strongly elliptic operator with real-analytic coefficients in $\bar{\Omega}$, so by known theorems on elliptic regularity (see [4], p. 336 and [5], p. 205), it follows that $\psi$ extends as a real-analytic function across $\partial \Omega$, still satisfying $L \psi=0$. According to equation (25), the continuation of $\psi$ generates an anti-analytic extension of $J\left(z_{0}, \zeta\right)$ across $\partial \Omega$. Since $J(z, \zeta)=\overline{J(\zeta, z)}$, this proves the lemma.

From the representation by a kernel function given in Theorem 4, it is now easy to prove the regularity of the canonical divisor.

THeOREM 5. In the space $A^{p}$ for $1 \leq p<\infty$, the canonical divisor $G$ of each finite zero-set has an analytic continuation to a disk $|z|<R$ for some $R>1$, and $G(z) \neq 0$ there except for its prescribed zeros.

Proof. According to Theorem 4, the canonical divisor $G$ has the form (19), where $J(z, \zeta)$ is the kernel function of $A_{w}^{2}$ with $w=|B|^{p}$. By Lemma $9, J(z, 0)$ has an analytic continuation to a larger disk. Thus it remains only to show that $J(z, 0) \neq 0$ at all points $z$ on $\mathbb{T}$. But the representation shows that $G$ has a smooth extension to the 
closed disk, so it has the expansive property of Lemma 4. This and the continuity of $G$ up to the boundary show that $|G(z)| \geq 1$ on $\mathbb{T}$, as shown in Lemma 5 . Since $|B(z)|=1$ on $\mathbb{T}$, the representation of $G$ therefore shows that $J(z, 0) \neq 0$ on $\mathbb{T}$, and the proof is complete.

COROllaRY. The canonical divisor is unique even for $p=1$.

Proof. For finite zero-sets, this is a corollary of Theorem 4. For infinite zero-sets, one approximates the canonical divisor $G$ by the canonical divisor $G_{n}$ of a finite set, as in Lemma 7. Theorem 5 says that $G_{n}$ has a smooth extension to $\overline{\mathbb{D}}$, and so by Lemma 4 it has the expansive property. The proof of Theorem 2 now applies to show that $G$ is a contractive divisor. But there can be at most one contractive divisor. Indeed, if $H$ were another contractive divisor, then $\|H / G\|_{p} \leq\|H\|_{p}=1$ and similarly $\|G / H\|_{p} \leq 1$. Thus the nonvanishing function $f=G / H$ has the property $\|f\|_{p}^{p}+\|1 / f\|_{p}^{p} \leq 2$, which is impossible unless $f(z) \equiv e^{i \theta}$, a constant of modulus one. Alternatively, it can be argued (see $\S 7$ ) that the contractive property implies the extremal property which defines the canonical divisor. The uniqueness is then established as in the proof of Theorem 2.

It may be remarked that the existence of an analytic continuation in the case $2<p<\infty$ also follows directly from the result for $1<p \leq$ 2 , in exactly the same way as the result for all even integers follows from its truth for $p=2$.

7. Concluding remarks. Let us now observe that for $1 \leq p<\infty$, the Bergman space $A^{p}$ has no isometric zero-divisors. Since the contractive divisor is unique (under the normalization $G(0)>0$ ), it is the only possible candidate for an isometric zero-divisor. If $\|G f\|_{p}=$ $\|f\|_{p}$ for all $f \in A^{p}$, then in particular $\left\|G^{n}\right\|_{p}=\|G\|_{p}=1$ for $n=1,2, \ldots$ But $|G(z)|>1$ on a set of positive area, so this is impossible.

In fact, it can be shown that $\|G f\|_{p}>\|f\|_{p}$ for all nonconstant functions $f \in A^{p}$, so the divisor $G$ is properly contractive except when applied to a constant multiple of itself. For canonical divisors $G$ of finite zero-sets, it is implicit in the proof of Lemma 4 that

$$
\iint_{\mathbb{D}}\left(|G|^{p}-1\right)|f|^{p} d \sigma=\iint_{\mathbb{D}} \varphi \Delta\left(|f|^{p}\right) d \sigma>0
$$

for all nonconstant functions $f$ which are analytic in $\overline{\mathbb{D}}$. In view of 
the representation (9), this formula may be written as

$$
\begin{aligned}
& \iint_{\mathbb{D}}\left(|G|^{p}-1\right)|f|^{p} d \sigma \\
& \quad=\pi \iint_{\mathbb{D}} \iint_{\mathbb{D}} \Gamma(z, \zeta) \Delta\left(|G(z)|^{p}\right) \Delta\left(|f(\zeta)|^{p}\right) d \sigma(z) d \sigma(\zeta),
\end{aligned}
$$

where $\Gamma(z, \zeta)$ is the biharmonic Green function of $\mathbb{D}$. In a later paper, it will be shown that this last formula remains valid under very general hypotheses, allowing in particular an arbitrary canonical divisor $G$ and an arbitrary function $f \in A^{p}$. An immediate consequence is that $\|G f\|_{p}>\|f\|_{p}$ if $f$ is any nonconstant function in $A^{p}$.

It should be noted that the extremal property used to define the canonical divisor actually follows directly from the contractive property. Indeed, let $g$ be a contractive divisor in $A^{p}$ for a zero-set $\left\{\zeta_{j}\right\}$ in $\mathbb{D} \backslash\{0\}$. Then for any function $f \in N^{p}$,

$$
|f(0) / g(0)|<\|f / g\|_{p} \leq\|f\|_{p}
$$

unless $f$ is a constant multiple of $g$. In particular, if $\|f\|_{p} \leq 1$ and $f$ vanishes on $\left\{\zeta_{j}\right\}$, then $|f(0)|<|g(0)|$ unless $f$ is a constant multiple of $g$.

As Hedenmalm [8] has already observed in the case $p=2$, the results of this paper extend mutatis mutandis to zero-sets containing the origin. The canonical divisor is then defined to maximize $\left|g^{(m)}(0)\right|$ among all $g \in N^{p}$ with $\|g\|_{p}=1$, where $m$ is the prescribed order of the zero at the origin. It is again a contractive zero-divisor in $A^{p}$.

With some logical restructuring, the methods of this paper can be used to extend the contractive property to $0<p<1$. For instance, the representation (19) in terms of a kernel function can then be taken as a definition of the canonical divisor for a finite zero-set, and it can be shown to have the contractive property. Also, the function $G_{\alpha}$ defined by (16) can be verified still to have the orthogonality property of Lemma 1 , so it is the contractive divisor for the single point $\alpha$. The details are omitted, but in a later paper the results will be developed in a more general context for $0<p<\infty$. In fact, the entire scheme of contractive divisors can be carried out in Bergman spaces $A^{p}(\Omega)$ over more general Jordan domains $\Omega$ with analytic boundary, as indicated in the introduction. Some of the results extend to arbitrary invariant subspaces. These generalizations will be described in the later paper.

One further consequence of Theorem 5 , on the analytic continuation of the canonical divisor $G$ of a finite zero-set, should be noted. Let $1 \leq p<\infty$ and fix a zero-set $\left\{\zeta_{j}\right\}, j=1,2, \ldots, n$. Assume 
for notational simplicity that all $\zeta_{j}$ 's are distinct. Then by a standard application of the duality relation (cf. [3], p. 110),

$$
G(0)=\inf \|1-f\|_{q},
$$

where $q=p /(p-1)$ is the conjugate index and the infimum is taken over all functions $f \in L^{q}$ which annihilate $N^{p}$. A unique extremal function $F$ exists for this dual extremal problem, and it has the form

$$
F(z)=\sum_{j=1}^{n} c_{j}\left(1-\zeta_{j} \bar{z}\right)^{-2}+h,
$$

where the $c_{j}$ 's are complex constants and $h$ is an $L^{q}$ function which annihilates $A^{p}$. (Thus by Havin's lemma, $h$ is in the $L^{q}$-closure of the functions $\partial \psi / \partial \bar{z}$, where $\psi \in C_{0}^{\infty}(\mathbb{D})$.) The extremality of $G$ and $F$ and the conditions for equality in Hölder's inequality now imply (for $p>1$ )

$$
G(z)[1-F(z)] \geq 0 \quad \text { a.e. }
$$

and

$$
|G(z)|^{p}=C|1-F(z)|^{q} \quad \text { a.e. }
$$

where $C=G(0)^{-q}$ is a positive constant. In view of Theorem 5 , we have arrived at the following result.

Theorem 6. For $1 \leq p<\infty$, let $N^{p}$ be the set of functions in the Bergman space $A^{p}$ which vanish on a prescribed finite set of points $\zeta_{j}$ in $\mathbb{D}$, with prescribed multiplicities. Let $F$ be the (unique) function which minimizes $\|1-f\|_{q}$ among all $f \in L^{q}$ which annihilate $N^{p}$, where $q$ is the conjugate index. Then $F$ extends to a larger disk as a function which is real-analytic except possibly at the points $\zeta_{j}$.

Finally, we want to pose a question. If $G$ is the canonical divisor of an infinite $A^{p}$ zero-set, and if the zero-set has a positive distance from some open arc of the unit circle, must $G$ have an analytic continuation over this arc? For $p=2$ this follows from a theorem of Akutowicz and Carleson [1], who treated other Hilbert spaces as well. Simpler proofs and generalizations can be found in Shapiro [15]. For $H^{p}$ spaces the zero-divisors are Blaschke products, which do have the stated continuation property, but for Bergman spaces the case of general $p$ seems to remain open.

Note added in proof. Daniel Luecking and Krzysztof Samotij have independently pointed out to us that the uniqueness of the canonical divisor for $p=1$ is a simple consequence of the strict convexity of $A^{1}:\|f\|_{1}=\|g\|_{1}=1$ implies $\|f+g\|_{1}<2$ unless $f=g$. 


\section{REFERENCES}

[1] E. J. Akutowicz and L. Carleson, The analytic continuation of interpolatory functions, J. Analyse Math., 7 (1959-60), 223-247.

[2] N. Dunford and J. T. Schwartz, Linear Operators, Part I, Interscience, New York, 1958; Wiley reprint, 1988.

[3] P. L. Duren, Theory of $H^{p}$ spaces, Academic Press, New York, 1970.

[4] G. B. Folland, Introduction to Partial Differential Equations, Princeton University Press, Princeton, N.J., 1976.

[5] A. Friedman, Partial Differential Equations, Holt, Rinehart and Winston, New York, 1969.

[6] P. R. Garabedian, A partial differential equation arising in conformal mapping, Pacific J. Math., 1 (1951), 485-524.

[7] _ Partial Differential Equations, Second Edition, Chelsea Publ. Co., New York, 1986.

[8] H. Hedenmalm, A factorization theorem for square area-integrable analytic functions, J. Reine Angew. Math., 422 (1991), 45-68.

[9] C. Horowitz, Zeros of functions in the Bergman spaces, Duke Math. J., 41 (1974), 693-710.

[10] G. Köthe, Topological Vector Spaces, I, Second Printing, Springer-Verlag, BerlinHeidelberg-New York, 1983.

[11] Z. Nehari, Conformal Mapping, McGraw-Hill, New York, 1952; Dover reprint, 1975.

[12] K. Yu. Osipenko and M. I. Stessin, On recovery problems in Hardy and Bergman spaces, Mat. Zametki, 49 (1991), No. 4, 95-104 (Russian).

[13] J. Ralston and L. Sario, A relation between biharmonic Green's functions of simply supported and clamped bodies, Nagoya Math. J., 61 (1976), 59-71.

[14] W. Rudin, Functional Analysis, McGraw-Hill, New York, 1973.

[15] H. S. Shapiro, Overconvergence of sequences of rational functions with sparse poles, Ark. Mat., 7 (1968), 343-349.

[16] _- Topics in Approximation Theory, Lecture Notes in Math. No. 187, Springer-Verlag, Berlin-Heidelberg-New York, 1971.

[17] _ The Schwarz Function and its Generalization to Higher Dimensions, Univ. of Arkansas Lecture Notes in Math. Sciences Vol. 9, John Wiley \& Sons, New York, 1992.

Received July 2, 1991. The first and second authors were supported in part by grants from the National Science Foundation.

UNIVERSITY OF MICHIGAN

ANN ARBor, MI 48109

UNIVERSITY OF ARKANSAS

FAYETTEVILLE, AR 72701

Royal INSTitute of TeChNOLOGY

S-100 44 STOCKHOLM, SwedeN

SWEDEN

AND

UNIVERSITY OF TENNESSEE

KNOXVILLE, TN 37916 


\title{
PACIFIC JOURNAL OF MATHEMATICS
}

Founded by

\author{
E. F. Beckenbach (1906-1982) F. Wolf (1904-1989)
}

\section{EDITORS}

V. S. VARADARAJAN

(Managing Editor)

University of California

Los Angeles, CA 90024-1555

vsv@math.ucla.edu

Herbert Clemens

University of Utah

Salt Lake City, UT 84112

clemens@math.utah.edu

F. Michael Christ

University of California

Los Angeles, CA 90024-1555

christ@math.ucla.edu

THOMAS ENRIGHT

University of California, San Diego

La Jolla, CA 92093

tenright@ucsd.edu
Nicholas ERcolani

University of Arizona

Tucson, AZ 85721

ercolani@math.arizona.edu

R. FInN

Stanford University

Stanford, CA 94305

finn@gauss.stanford.edu

VAughan F. R. Jones

University of California

Berkeley, CA 94720

vfr@math.berkeley.edu

STEVEN KERCKHOFF

Stanford University

Stanford, CA 94305

spk@gauss.stanford.edu

\section{C. MOORE}

University of California

Berkeley, CA 94720

MARTIN SCHARLEMANN

University of California

Santa Barbara, CA 93106

mgscharl@henri.ucsb.edu

Harold Stark

University of California, San Diego La Jolla, CA 92093

\section{SUPPORTING INSTITUTIONS}

UNIVERSITY OF ARIZONA

UNIVERSITY OF BRITISH COLUMBIA

CALIFORNIA INSTITUTE OF TECHNOLOGY

UNIVERSITY OF CALIFORNIA

MONTANA STATE UNIVERSITY

UNIVERSITY OF NEVADA, RENO

NEW MEXICO STATE UNIVERSITY

OREGON STATE UNIVERSITY
UNIVERSITY OF OREGON

UNIVERSITY OF SOUTHERN CALIFORNIA

STANFORD UNIVERSITY

UNIVERSITY OF HAWAII

UNIVERSITY OF TOKYO

UNIVERSITY OF UTAH

WASHINGTON STATE UNIVERSITY

UNIVERSITY OF WASHINGTON 


\section{PACIFIC JOURNAL OF MATHEMATICS}

Volume $157 \quad$ No. $1 \quad$ January 1993

Permutation enumeration symmetric functions, and unimodality

FRANCESCO BRENTI

On the analytic reflection of a minimal surface

JAIGYOUNG CHOE

Contractive zero-divisors in Bergman spaces

Peter Larkin DUREn, DMitry Khavinson, Harold SEymour

SHAPIRO and CARL SUNDBERG

On the ideal structure of positive, eventually compact linear operators on

Banach lattices

RUEY-JEN JANG and HAROLD DEAN VICTORY, JR.

A note on the set of periods for Klein bottle maps

JAUME LLIBRE

Asymptotic expansion at a corner for the capillary problem: the singular case

\section{ERICH MIERSEMANN}

A state model for the multivariable Alexander polynomial

JUN MURAKAMI

Free Banach-Lie algebras, couniversal Banach-Lie groups, and more

Vladimir G. Pestov

Four manifold topology and groups of polynomial growth

RICHARD ANDREW STONG

A remark on Leray's inequality

AKIRA TAKESHITA

$A_{\infty}$ and the Green function

JANG-MEI GLORIA WU

Integral spinor norms in dyadic local fields. I 